\title{
Hemorrhagic plaques in the oral cavity: A clue to diagnosing thrombocytopenia
}

\section{Aswathi Raj, Amina Asfiya, Malcolm Pinto, Manjunath Shenoy M, Spandana P Hegde, Vishal Bejai, Ashmiya Abdul Razak}

Department of Dermatology, Yenepoya Medical College, Deralakatte, Mangalore-575018, India

Corresponding author: Dr. Aswathi Raj, E-mail: aswathirbr76@gmail.com

\begin{abstract}
Idiopathic thrombocytopenic purpura is a disorder with a myriad of possible clinical presentations. The mechanism of thrombocytopenia involves both increased platelet destruction and impaired platelet production. The patient can manifest a wide range of symptoms: from asymptomatic or minimal gingival bleeding to profuse bleeding from any site. The disease may first present itself to the dermatologist in cutaneous findings such as petechiae, purpura, and mucosal manifestations in the form of gingival bleeding and hemorrhagic bullae. The diagnosis of idiopathic thrombocytopenic purpura is mostly done by exclusion. In this report, we present two cases with characteristic oral manifestations, who were diagnosed, on investigation, with idiopathic thrombocytopenic purpura. The patients were successfully treated with immunosuppressive therapy. The report aims to raise awareness that would help in enabling prompt referral to the appropriate specialty, especially because of the rarity of this presentation.
\end{abstract}

Key words: Thrombocytopenia; Purpura; Oral cavity

\section{INTRODUCTION}

Idiopathic thrombocytopenic purpura (ITP) is a disorder characterized by a low platelet count (thrombocytopenia), usually of no known cause (idiopathic). As most causes appear to be related to antibodies against platelets, it is also known as immune thrombocytopenic purpura. Although most cases are asymptomatic, a significantly low platelet count can lead to bleeding diathesis and purpura. A platelet count below $50,000 \mathrm{~mm}^{3}$ increases risk of dangerous bleeding from trauma [1].

\section{CASE REPORT}

A 52-year-old female presented herself to the dermatology department with asymptomatic oral ulcers for fifteen days prior. On further probing, the patient admitted to having noticed multiple asymptomatic flat and red lesions over both legs for ten days prior.
She also had associated low-grade fever, constitutional symptoms, and reduced appetite and sleep for two weeks prior. There was no history of prior drug intake or associated gingival bleeding, headaches, blurring of vision, seizures, abdominal pain, nasal bleeding, hematuria, hematochezia, or melena. The family history was insignificant. She was a known case of hypothyroidism and hypertension under treatment. A general physical examination revealed no abnormality except for pallor. A systemic examination was normal. A dermatological examination revealed multiple nonpalpable, non-blanchable purpura over the bilateral lower limbs till the lower third, a few scattered lesions over the upper left half and abdomen, and an isolated ecchymotic patch over the left shoulder (Figs. 1 and 2). An examination of the oral cavity revealed multiple ulcers with surrounding hemorrhagic plaques over the buccal mucosa (Fig. 3). The patient was promptly referred to a physician for further evaluation. Laboratory investigations showed hemoglobin at $13.9 \mathrm{~g} / \mathrm{dL}$ and the

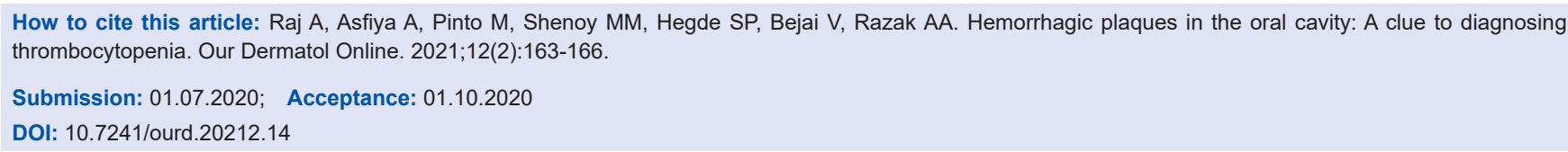




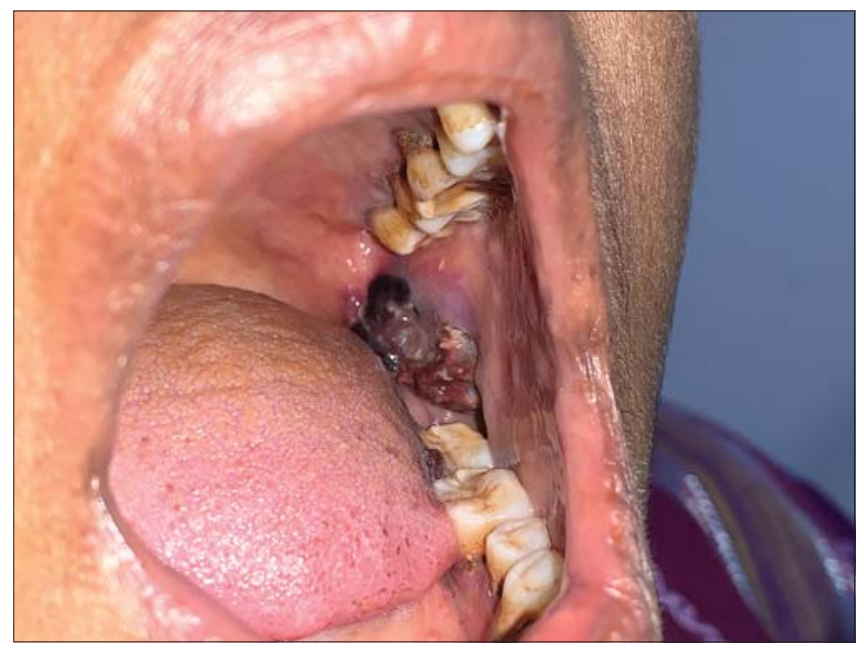

Figure 1: Hemorrhagic plaque on the left side of the buccal mucosa.

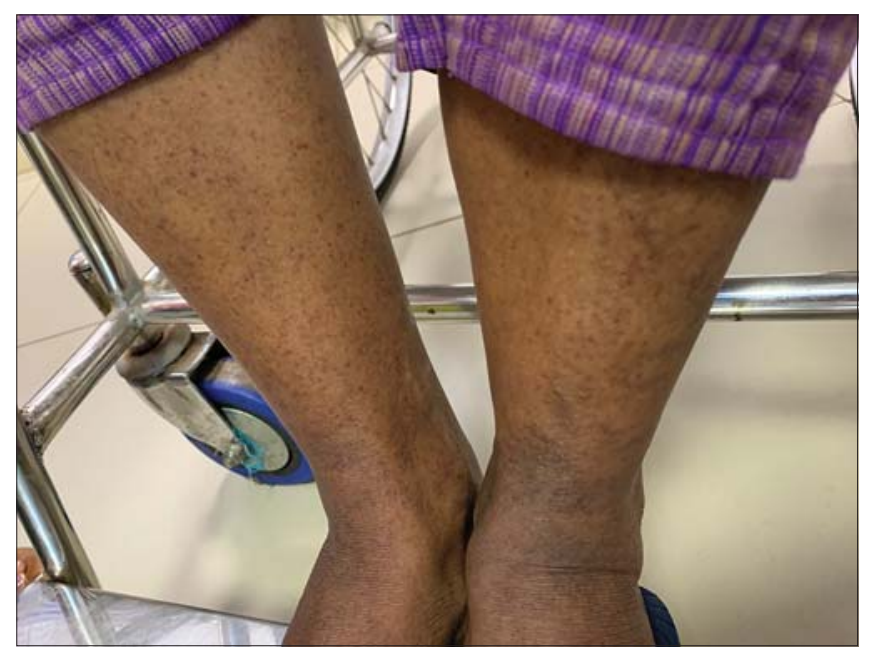

Figure 2: Purpura over the bilateral legs.

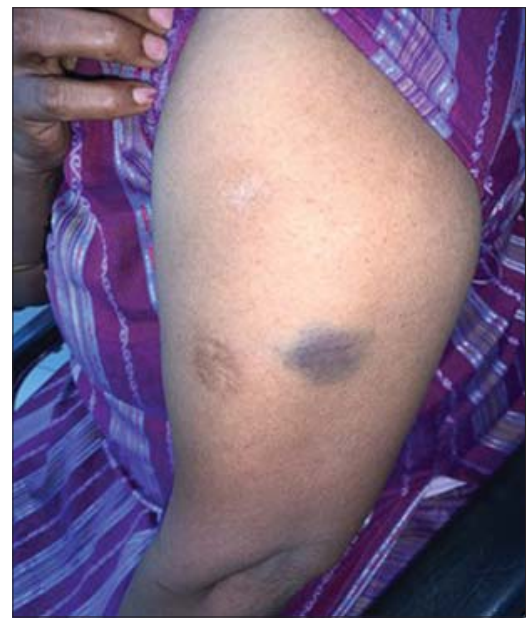

Figure 3: Ecchymosis over the left arm.

platelet count at $6000 / \mu \mathrm{L}$. The coagulation profile was normal. Tests for malaria and dengue were negative. Peripheral smear showed severe thrombocytopenia. The patient was, thus, diagnosed with ITP and initiated on antibiotics and steroids. However, due to a minimal response, hematologist opinion was sought and the patient as started on azathioprine $50 \mathrm{mg}$ BD along with a tapering dose of steroids. Following this, the platelet count increased and the cutaneous lesions improved.

The second patient was a 36-year-old male who presented himself with an asymptomatic dark lesion in the oral cavity and the right side of the tongue for fifteen days prior. There were no red lesions anywhere else on the body. No history of fever, weight loss, drug intake, joint pain was present, as well as no history suggestive of bleeding manifestations. No history of headache, blurring of vision, seizures, or other comorbidities was present either. An examination revealed normal vitals. An examination of the oral cavity revealed a hemorrhagic plaque over the posterior part of the right buccal mucosa and a smaller plaque over the right lateral border of the tongue (Figs. 4 and 5). The patient's general condition as well as a systemic examination were normal. A laboratory investigation revealed hemoglobin at $12.8 \mathrm{~g} / \mathrm{dL}$, the platelet count at $23000 / \mu \mathrm{L}$, the total count at $8200 / \mu \mathrm{L}$, and ESR at $9 \mathrm{~mm} / \mathrm{h}$. The coagulation profile was normal. Peripheral smear showed thrombocytopenia. The patient was started on oral prednisolone in tapering doses, considering a diagnosis of ITP. Over a period of two weeks, improvement in the platelet count was noted with a resolution of oral lesions.

\section{DISCUSSION}

ITP, also known as Werlhof's disease, is caused by antibody and cell-mediated platelet destruction and suppression of platelet production, which may occur without any predisposing factor (primary ITP) or secondary due to other conditions, including autoimmune disorders, neoplasms, congenital immune deficiencies, drugs, and infections. There are two clinical subtypes of primary ITP: acute primary ITP (present for less than 6 months), a self-limited form commonly seen in children subsequent to viral infections, and chronic primary ITP (present for more than 6 months), more commonly seen in young adult females between 20 to 40 years of age. Chronic ITP is characterized by antiplatelet antibodies directed against platelet membrane glycoproteins IIb/IIIa or Ib/IX complexes. The diagnostic criteria of ITP, outlined by an international working group, include either a confirmed platelet count of $\geq 50 \times 10^{9} / \mathrm{L}$ and $<100 \times 10^{9} / \mathrm{L}$ on two or more consecutive occasions 


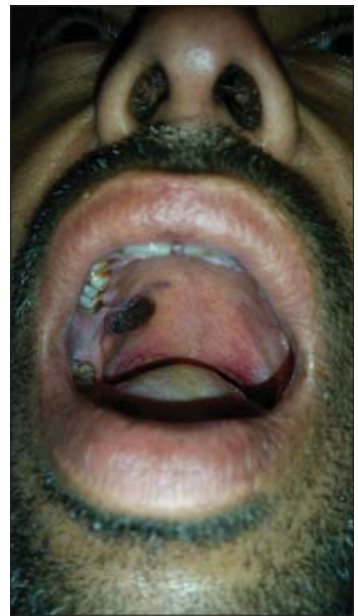

Figure 4: Hemorrhagic plaque over the posterior part of the oral cavity.

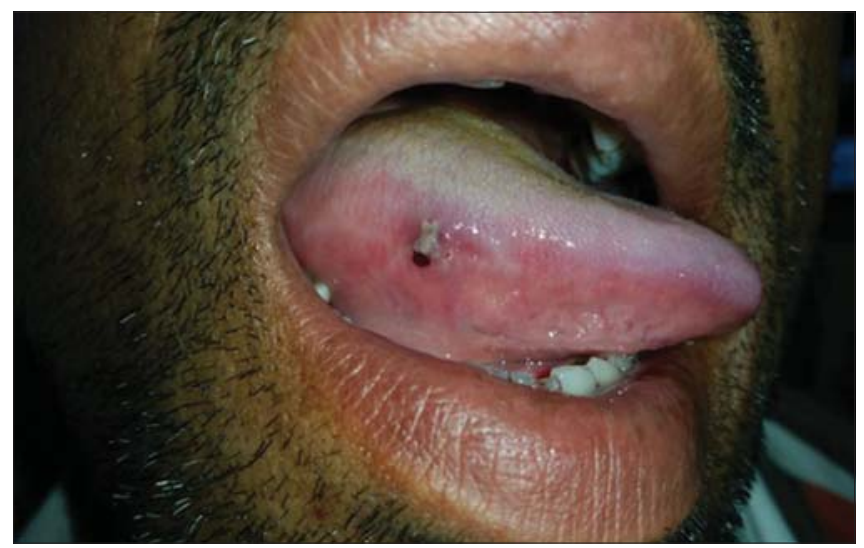

Figure 5: Hemorrhagic plaque over the right lateral side of the tongue.

over a period of one month or a confirmed platelet count of $<50 \times 10^{9} / \mathrm{L}$ on two or more consecutive occasions over any period of time; with normal hemoglobin, a normal white blood cell (WBC) count, no splenomegaly, normal peripheral smear, except for a low platelet count, and no evidence of an alternative nonautoimmune etiology of thrombocytopenia [2].

The first sign of thrombocytopenia is usually spontaneous bleeding or bleeding on minor trauma, as during brushing or flossing. Common sites involved are the lateral border of the tongue, the buccal mucosa, and the junction between the hard and soft palate. Clinical features include petechiae, purpura, ecchymosis, hematoma, and multiple hemorrhagic blisters [3]. The characteristic clinical indicators of ITP include easy bruising of the skin, mucocutaneous lesions such as petechiae and ecchymosis, epistaxis, gingival bleeding, and hemorrhagic bullae in the mouth [4]. Other symptoms may include hematemesis, menorrhagia, conjunctival hemorrhages, and melena. Symptomatic bleeding is uncommon unless the ITP is severe (platelet count of less than $30,000 / \mu \mathrm{L}$ ).
A platelet count below $10,000 / \mu \mathrm{L}$ is increases risk of internal bleed [5]. The disease is usually self-limiting, mostly occurring after a viral infection or immunization. Recovery is generally observed within six months. Some patients will develop chronic thrombocytopenia and usually die from cerebral hemorrhage. A similar case was reported by Sugiura T et al. in which a 79-year-old female presented herself with a sudden onset of gingival bleeding and hemorrhagic bullae on the buccal mucosa, with a platelet count of $2000 / \mu \mathrm{L}$ [5]. Another report describes a patient with multiple comorbidities who presented themself with bleeding from the gingiva in the lower anterior region with purpuric spots over the neck, forearms, right arm, dorsum of the tongue, hard palate, and maxillary tuberosity region with a platelet count of $6000 / \mu \mathrm{L}$ [1]. Our patient had no evidence of gingival bleeding, in contrast to most previously reported cases, making it important to examine the entire oral cavity for hemorrhagic lesions as this may sometimes be the only manifestation of the disease. Dermatologists should be aware of this unusual clinical finding to promptly institute treatment and prevention of any serious complications that may arise if the disease is left untreated.

\section{CONCLUSION}

ITP presenting itself as hemorrhagic bullae in the oral cavity is a rare finding. Very few cases have been reported in the medical literature with oral hemorrhage as the first symptom of ITP. Hence, it is imperative that dermatologists exercise a pragmatic approach to oral hemorrhage and consider ITP as a differential diagnosis in such cases. Prompt referral and treatment of these cases have the potential to lead to a significant reduction in mortality.

\section{Consent}

The examination of the patient was conducted according to the principles of the Declaration of Helsinki.

The authors certify that they have obtained all appropriate patient consent forms, in which the patients gave their consent for images and other clinical information to be included in the journal. The patients understand that their names and initials will not be published and due effort will be made to conceal their identity, but that anonymity cannot be guaranteed.

\section{REFERENCES}

1. Kayal L, Jayachandran S, Singh K. Idiopathic thrombocytopenic purpura. Contemp Clin Dent. 2014;5:410-4.

2. Rodeghiero F, Stasi R, Gernsheimer T, Michel M, Provan D, 


\section{www.odermatol.com}

Arnold DM, et al. Standardization of terminology, definitions and outcome criteria in immune thrombocytopenic purpura of adults and children: report from an international working group. Blood. 2009;113:2386-93.

3. Vasconcelos RB, Vasconcelos GB, do Nascimento MG, de Andrade Amorim VCS. Childhood idiopathic thrombocytopenic purpura: Oral manifestations and dental dare. Int J Oral Dent Health. 2018;4:067.

4. Sangwan A, Tewari S, Narula SC, Sharma RK, Sangwan P. Significance of periodontal health in primary immune thrombocytopenia: A case report and review of literature. J Dent
(Tehran). 2013;10:197-202.

5. Sugiura T, Yamamoto K, Murakami K, Horita S, Matsusue Y, Nakashima C, et al. Immune thrombocytopenic purpura detected with oral hemorrhage: A case report. J Dent (Shiraz). 2018;19:159-63.

Copyright by Aswathi Raj, et al. This is an open-access article distributed under the terms of the Creative Commons Attribution License, which permits unrestricted use, distribution, and reproduction in any medium, provided the original author and source are credited.

Source of Support: Nil, Conflict of Interest: None declared. 